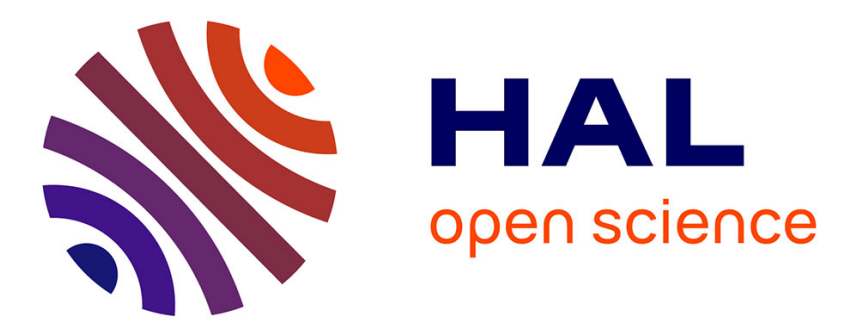

\title{
Online frequency band estimation and change-point detection
}

Zoran Tiganj, Mamadou Mboup, Sylvain Chevallier, Emmanuel Kalunga

\section{To cite this version:}

Zoran Tiganj, Mamadou Mboup, Sylvain Chevallier, Emmanuel Kalunga. Online frequency band estimation and change-point detection. 1st International Conference Systems and Computer Science, Aug 2012, Lille, France. hal-00756089

\section{HAL Id: hal-00756089 \\ https://hal.inria.fr/hal-00756089}

Submitted on 22 Nov 2012

HAL is a multi-disciplinary open access archive for the deposit and dissemination of scientific research documents, whether they are published or not. The documents may come from teaching and research institutions in France or abroad, or from public or private research centers.
L'archive ouverte pluridisciplinaire HAL, est destinée au dépôt et à la diffusion de documents scientifiques de niveau recherche, publiés ou non, émanant des établissements d'enseignement et de recherche français ou étrangers, des laboratoires publics ou privés. 


\title{
Online frequency band estimation and change-point detection
}

\author{
Zoran Tiganj*, Mamadou Mboup ${ }^{\dagger}$, Sylvain Chevallier* and Emmanuel Kalunga*‡ \\ ${ }^{*}$ LISV, University of Versailles, Velizy, France \\ $\dagger$ CReSTIC, University of Reims Champagne Ardenne, Reims, France \\ \& Non-A, INRIA Lille - Nord Europe, Villeneuve d'Ascq, France \\ $\ddagger$ FSATI, Tshwane University of Technology, Pretoria, South Africa
}

\begin{abstract}
Detecting change-points in time series is at the heart of numerous applications, as abrupt changes in signal properties are quite common in natural and industrial processes. In this paper we propose an algorithm for a particular changepoint detection problem where the frequency band of the signal changes at some points in the time axis. Apart from detecting the change-points, the proposed algorithm is also able to estimate the frequency bands. The main idea of the algorithm is to consider a simple local bandlimited model to represent the input signal in each sliding time window. The local model consists of a sum of two exponentials that, in the frequency domain, reads as the transfer function of a second order bandpass filter. Relying on the operational calculus, we obtain an explicit estimation of the parameters, that indicate the cut-off frequencies associated to each time window. The implementation is done in discrete time domain where the cut-off frequencies are computed as a combination of outputs of several FIR filters, providing a low computational cost online estimation. Experimental results show the efficiency and the stability of this algorithm even in presence of a moderate amount of noise.
\end{abstract}

\section{INTRODUCTION}

Change-point is a term commonly used to refer to the points in time where the properties of otherwise stationary process abruptly change. Processes with abrupt changes are common in natural and industrial systems. To illustrate the change-point detection problem we mention electrocardiogram (ECG) as a good example, since it is characterized with specific deflections which differ significantly from the rest of the signal. Another example is electroencephalogram (EEG) during epileptic seizures, which contains so-called spike and wave waveforms.

Detection of such change-points stands as an important research problem. Depending on the particular application, sometimes the detection is done after the recoding is finished (offline detection), but often the detection is required during the recording (online detection). A large amount of literature is devoted to the problem, in its general form. We refer to [1] for an introductive presentation containing many application examples and also [2]-[7] for studies more related to the work presented here.

In this paper we address a very particular change-point detection problem. We consider a piecewise noisy input signal, such that each piece is characterized with several frequency components, belonging to some frequency band. The band changes from one piece to its neighbor so that two consecutive pieces always occupy different frequency bands. Here we propose an algorithm for online estimation of the cut-off frequencies of the bands. This also gives us an estimation of the time instants when the bands change.

Potential applications of such algorithm, same as for the change-point detection algorithms in general, are very diverse. They span form industrial to biomedical applications. For example, the epileptic spikes and wave complex mentioned above are characterized by an abrupt change in frequency. This change is assumed to be caused by sudden activation of a very large number of neurons whose synchronous cumulative activity brings new frequency components into the EEG spectrum [8]. Fast detection of epileptic attacks is important to reduce the possible consequences for the patient, thus the online performance of the algorithm is often required. Another example based on the EEG signal is related with braincomputer interface (BCI) applications, where so called event related desynchronization/synchronization (ERD/ERS) caused by imagination of a movement is used to give a command to the computer. The ERD/ERS phenomenon is also caused by simultaneous activation of large neural circuits in the motor cortex during imagination of a movement of some body part [9]. The manifestation of the phenomenon is again the change of several frequency components in the signal. Since BCI applications are always in real time, the online detection of ERD/ERS is required. Industrial applications include faults detection and diagnosis in electro-mechanic controlled plants [10], [11], in chemical processes [12], detection of structural damages [13], quality control [14], etc.

The main idea of the proposed algorithm is to consider a simple local bandlimited model to represent the input signal in each sliding time window of a given length. The local model consists of a sum of two exponentials which, in the frequency domain, reads as the transfer function of a second order bandpass filter. Two exponentials are enough to estimate the cut-off frequencies. Of course, we could use more than two, the price would be payed by increasing complexity of the algorithm. Relying on the operational calculus, we obtain an explicit estimation of the parameters that indicate the cutoff frequencies. To estimate them we take advantage of the algebraic framework of [15] (see also [16], [17], [18]). Finally we slide the time window along the signal and compute the cut-off frequencies for each time instance. This is in time 
domain realized using a convolution integral. The solution is implemented in discrete time domain as a combination of FIR filters, providing an efficient online algorithm.

The proposed algorithm is detailed in section II. first for a lowpass model. for which the discrete time implementation is shown, and then transferred into bandpass model. In section III results are shown on synthetic signals, ranging from low number of sine components to bandpass signal. We also provide a parameter analysis. The section IV concludes this paper.

\section{ESTIMATION OF THE CUT-OFF FREQUENCIES}

Let $x(t)$ denotes the unobserved noise-free signal: $x(t)=$ $y(t)-\varpi(t)$. Let $T>0$ be given. To any $\tau \geqslant 0$, we associate the interval $I_{\tau}^{T}=[\tau, \tau+T)$. Thus the restriction of the signal $x(t)$ in $I_{\tau}^{T}$ is defined as:

$$
x_{\tau}(t) \triangleq H(t) x(t+\tau), \quad t \in[0, T)
$$

We propose to model the input signal with a local model that consists of a sum of two exponentials which, in the frequency domain, reads as the transfer function of a second order bandpass filter. We consider a second order lowpass filter form, which will be transformed later into a bandpass form. Signal $x_{\tau}(t)$ reads in the operational domain as:

$$
x_{\tau}(s)=\frac{b_{0}(\tau)+b_{1}(\tau) s}{1-a_{1}(\tau) s-a_{2}(\tau) s^{2}}
$$

\section{A. Finding coefficients of the lowpass model}

To find the bandpass cut-off frequencies, only $a_{1}(\tau)$ and $a_{2}(\tau)$ must be characterized, as we will see later $b_{0}(\tau)$ and $b_{1}(\tau)$ are irrelevant for this problem. Thus we annihilate them by taking the second order operational derivative $d^{2} / d s^{2}$ of the equation (2). The equation then becomes:

$$
\begin{array}{r}
x_{\tau}(s)^{(2)}=2 a_{1}(\tau) x_{\tau}(s)^{(1)}+a_{1}(\tau) s x_{\tau}(s)^{(2)}+2 a_{2}(\tau) x_{\tau}(s) \\
+4 a_{2}(\tau) s x_{\tau}(s)^{(1)}+a_{2}(\tau) s^{2} x_{\tau}(s)^{(2)} .
\end{array}
$$

Since there are now two unknowns $\left(a_{1}(\tau)\right.$ and $\left.a_{2}(\tau)\right)$ and only one equation, we take another $d / d s$ to obtain a system of two equations. The second equation is:

$$
\begin{array}{r}
x_{\tau}(s)^{(3)}=3 a_{1}(\tau) x_{\tau}(s)^{(2)}+a_{1}(\tau) s x_{\tau}(s)^{(3)}+6 a_{2}(\tau) x_{\tau}(s)^{(1)} \\
+6 a_{2}(\tau) s x_{\tau}(s)^{(2)}+a_{2}(\tau) s^{2} x_{\tau}(s)^{(3)}
\end{array}
$$

By the very classical rules of operational calculus, multiplication by $s$ corresponds to time derivation: $s \hat{x}_{\tau} \bullet \frac{d}{d t} x_{\tau}(t)$. As numerical differentiation is difficult and ill-conditioned, equations (3) and (4) are divided by a $s^{\nu}$, for an integer $\nu>2$. Only negative powers of $s$, and hence integral operators, will intervene in the resulting equation.

The unobserved signal $x_{\tau}$ can now be replaced by its noisy observation counterpart $y_{\tau}$. Instead of $a_{1}(\tau)$ and $a_{2}(\tau)$, only their estimates $\tilde{a_{1}}(\tau)$ and $\tilde{a_{2}}(\tau)$ are now available.

The equations (3) and (4) can be reformulated as

$$
\hat{P} \tilde{\Theta}=\hat{Q}
$$

where $\tilde{\Theta}=\left[\begin{array}{c}\tilde{a_{1}}(\tau) \\ \tilde{a_{2}}(\tau)\end{array}\right]$ and:

$$
\begin{gathered}
\hat{P}=\left[\begin{array}{cc}
\frac{2 x_{\tau}(s)^{(1)}}{s^{\nu}}+\frac{x_{\tau}(s)^{(2)}}{s^{\nu-1}} & \frac{2 x_{\tau}(s)}{s^{\nu}}+\frac{4 x_{\tau}(s)^{(1)}}{s^{\nu-1}}+\frac{x_{\tau}(s)^{(2)}}{s^{\nu-2}} \\
\frac{3 x_{\tau}(s)}{s^{\nu}}+\frac{x_{\tau}(s)^{(3)}}{s^{\nu-1}} & \frac{6 x_{\tau}(s)^{(1)}}{s^{\nu}}+\frac{6 x_{\tau}(s)^{(2)}}{s^{\nu-1}}+\frac{x_{\tau}(s)^{(3)}}{s^{\nu-2}}
\end{array}\right], \\
\hat{Q}=\left[\begin{array}{c}
\frac{x_{\tau}(s)^{(2)}}{s^{\nu}} \\
\frac{x_{\tau}(s)^{(3)}}{s^{\nu}}
\end{array}\right] .
\end{gathered}
$$

\section{B. Expressing the system in the time domain}

The numerical estimates are now obtained by expressing the system (5) in the time domain. For this, let us first recall that differentiation with respect to $s$ corresponds to multiplication by $-t: \frac{d}{d s} \hat{x}_{\tau} \bullet-t x_{\tau}(t)$, and division by $s^{m}, m>0$ maps to the $m^{\text {th }}$-iterated time integration:

$$
s^{-m} \hat{x} \bullet \frac{1}{(m-1) !} \int_{0}^{t}(t-\alpha)^{m-1} x(\alpha) d \alpha .
$$

With these rules in mind and using integration by parts, the time domain analog of the system (5) is expressed as:

$$
P(t, \tau) \tilde{\Theta}=Q(t, \tau)
$$

where:

$$
\begin{gathered}
Q(t, \tau)=\left[\begin{array}{l}
d_{1} \\
d_{2}
\end{array}\right] \\
d_{1}=-\int_{0}^{t} \frac{(t-\tau)^{\nu-1} \tau^{2}}{(\nu-1) !} x_{\tau}(\lambda) d \lambda \\
d_{2}=\int_{0}^{t} \frac{(t-\tau)^{\nu-1} \tau^{3}}{(\nu-1) !} x_{\tau}(\lambda) d \lambda \\
P(t, \tau)=\left[\begin{array}{ll}
d_{3} & d_{4} \\
d_{5} & d_{6}
\end{array}\right] \\
d_{3}=\int_{0}^{t}\left(\frac{2(t-\lambda)^{\nu-1} \lambda}{(\nu-1) !}-\frac{(t-\lambda)^{\nu-2} \lambda^{2}}{(\nu-2) !}\right) x_{\tau}(\lambda) d \lambda
\end{gathered}
$$

$$
d_{4}=\int_{0}^{t}\left(\frac{-2(t-\lambda)^{\nu-1}}{(\nu-1) !}+\frac{4(t-\lambda)^{\nu-2} \lambda}{(\nu-2) !}-\frac{(t-\lambda)^{\nu-3} \lambda^{2}}{(\nu-3) !}\right) x_{\tau}(\lambda) d \lambda
$$

$$
d_{5}=\int_{0}^{t}\left(\frac{-3(t-\lambda)^{\nu-1} \lambda^{2}}{(\nu-1) !}+\frac{(t-\lambda)^{\nu-2} \lambda^{3}}{(\nu-2) !}\right) x_{\tau}(\lambda) d \lambda
$$

$d_{6}=\int_{0}^{t}\left(\frac{6(t-\lambda)^{\nu-1} \lambda}{(\nu-1) !}-\frac{6(t-\lambda)^{\nu-2} \lambda^{2}}{(\nu-2) !}+\frac{(t-\lambda)^{\nu-3} \lambda^{3}}{(\nu-3) !}\right) x_{\tau}(\lambda) d \lambda$.

Redefining the intervals $I_{\tau}^{T}$ by their causal counterparts, $I_{\tau}^{T} \triangleq[\tau-T, \tau]$, the system from equation (6) is considered for $t=T$ and with the change of variable $\lambda \rightarrow T-\lambda$. By defining

$$
\begin{gathered}
h_{1}(\lambda)=-T^{2}(1-\lambda)^{2} \lambda^{\nu-1} \\
h_{2}(\lambda)=T^{3}(1-\lambda)^{3} \lambda^{\nu-1} \\
h_{3}(\lambda)=2 T\left((1-\lambda) \lambda^{\nu-1}-(1-\lambda)^{2} \lambda^{\nu-2}(\nu-1)\right) \\
h_{4}(\lambda)=-2 \lambda^{\nu-1}+4(1-\lambda) \lambda^{\nu-2}(\nu-1) \\
-(1-\lambda)^{2} \lambda^{\nu-3}(\nu-1)(\nu-2) \\
h_{5}(\lambda)=T^{2}\left(-3(1-\lambda)^{2} \lambda^{\nu-1}+(1-\lambda)^{3} \lambda^{\nu-2}(\nu-1)\right) \\
h_{6}(\lambda)=6 T(1-\lambda) \lambda^{\nu-1}-6 T(1-\lambda)^{2} \lambda^{\nu-2}(\nu-1) . \\
+T(1-\lambda)^{3} \lambda^{\nu-3}(\nu-1)(\nu-2)
\end{gathered}
$$

the elements of the coefficients $\hat{P}$ and $\hat{Q}$ could be rewritten as convolution integrals:

$$
d_{i}=\int_{0}^{\infty} h_{i}(\lambda) x(t-\lambda) d \lambda
$$

where $i=1, \ldots, 6$. 


\section{Discrete-time implementation}

We turn now to the discrete-time setting where only time samples of the observation, $y_{m}=y\left(t_{m}\right)$, are available. Assuming a uniform sampling, let $t_{m}=m T_{s}, m=0,1, \ldots$, with a sampling period $T_{s}$. Let $T=M T_{s}$ and $\nu>2$ be given. Set $\tau=n T_{s}$ and compute the finite sequence for $i=1,2, \ldots, 6$ $\left\{h_{i, m} \triangleq h_{i}\left(t_{m}\right)\right\}_{m=0}^{M}$. Then, by a numerical integration method with abscissas $t_{m}$ and weights $W_{m}, m=0, \cdots, M$, we obtain the approximation of $d_{1}, d_{2}, d_{3}, d_{4}, d_{5}$ and $d_{6}$.

$$
d_{i}(\tau) \approx d_{i, n}=\sum_{m=0}^{M} g_{i, m} y_{n-m}
$$

where

$$
g_{i, m} \triangleq W_{m} h_{i, m}
$$

In all the sequel, we use the trapezoidal method for which $W_{0}=W_{M}=0.5$, and $W_{m}=1, m=2, \cdots, M-1$. As $\tau=\tau_{n}=n T_{s}$ varies (sliding windows $I_{n}^{M}$ ), $d_{1}, d_{2}, d_{3}, d_{4}$, $d_{5}$ and $d_{6}$ read as the outputs of the FIR filters, with impulse response sequences $\left\{g_{i, m}\right\}_{m=0}^{M}$ for $i=1,2, \ldots, 6$. Accordingly, solving the system from equation (6) we obtain discrete approximations of $a_{1, n} \approx a_{1}\left(n T_{s}\right)$ and $a_{2, n} \approx a_{2}\left(n T_{s}\right)$.

\section{Transferring lowpass into bandpass model}

The general bandpass form is obtained by transferring the low-pass general form:

$$
h(s)=\frac{b(\tau)}{a(\tau)+s}
$$

into band-pass:

$$
h(h 1(s))=\frac{b(\tau)}{a(\tau)+\frac{s^{2}+\omega_{1}(\tau) \omega_{2}(\tau)}{\left(\omega_{2}(\tau)-\omega_{1}(\tau)\right) s} .}
$$

Equation (8) can be written in similar form as equation (2):

$$
h(h 1(s))=\frac{\frac{\left(\omega_{2}(\tau)-\omega_{1}(\tau)\right) b(\tau) s}{\omega_{1}(\tau) \omega_{2}(\tau)}}{1+\frac{\left(\omega_{2}(\tau)-\omega_{1}(\tau)\right)}{\omega_{1}(\tau) \omega_{2}(\tau)} a(\tau) s+\frac{1}{\omega_{1}(\tau) \omega_{2}(\tau)} s^{2}} .
$$

Now we chose $a(\tau)=1$ and from here $\tilde{\omega}_{1, n}$ and $\tilde{\omega}_{2, n}$, the discrete approximations of $\tilde{\omega}_{1}(\tau)$ and $\tilde{\omega}_{2}(\tau)$, can be expressed as:

$$
\begin{array}{r}
\tilde{a}_{2, n} \tilde{\omega}_{2, n}^{2}-\tilde{a}_{1, n} \tilde{\omega}_{2, n}+1=0, \\
\tilde{\omega}_{1, n}=\frac{-1}{\tilde{a}_{2, n} \tilde{\omega}_{2, n}} .
\end{array}
$$

Thus for each $n$ (as $I_{n}^{M}$ slides), the cut-off frequencies of the model $\tilde{\omega}_{1, n}$ and $\tilde{\omega}_{2, n}$ can be estimated. Since $\tilde{\omega}_{2, n}$ is a solution of a square equation and $\tilde{\omega}_{1, n}$ is computed using $\tilde{\omega}_{2, n}$, we will have two solutions for each estimate of the cut-off frequencies.

\section{RESULTS}

This section shows the results obtained for different signal complexity, ranging from few sine functions to bandpass signal, and proposes a parameter analysis of the algorithm. The evaluations are always performed on signals composed of three pieces, each one having different spectral properties. The length of the signal was 90s with sampling frequency of $100 \mathrm{~Hz}$ giving 9000 samples. We assess the ability of the algorithm to accurately estimate the frequency band occupied by each piece of the signal.

In the simulations to follow, the estimated values of both solutions for $\tilde{\omega}_{1, n}$ and $\tilde{\omega}_{2, n}$ are first processed by a median filter (the length of the median filter was set to 35 samples) and then smoothed by a moving average filter (the length of the moving average filter was set to 45 samples). Such processing was necessary to reduce the perturbations of the estimations caused mostly by the numerical instability of the matrix inverse $P^{-1}$. The lengths of the two filters were chosen experimentally, by observing the stability of the results. Parameter $\nu$ was set to 3 and parameter $M$ was set to 10 . The influence of these values will be discussed later.

The first test signal (top row of Fig. 1) is composed only from two sine functions in each of the three pieces. Each piece displays different frequencies for the sine functions, as shown in the middle row of Fig. 1 (here and on the remaining figures, each frequency plot refers to the corresponding part of the signal). Such signal is well suited for the second order model used in this algorithm. Both solutions for $\tilde{\omega}_{1, n}$ and $\tilde{\omega}_{2, n}$ are given on the plot in the bottom row of Fig. 1. Even though they are not fully stable, they indicate well the spectral structure of the corresponding parts of the signal.

Figure 2 shows the estimation obtained for an input signal consisting in a sum of 5000 sinusoids, each with random phase and amplitude. The signal is again composed of three pieces: for the first one the frequency components were randomly drawn from the interval $15-18 \mathrm{~Hz}$, for the second from the interval $12-15 \mathrm{~Hz}$ and for the third from the interval $9-$ $12 \mathrm{~Hz}$. The oscillations of the cut-off frequency estimations are now larger due to the very high degree of undermodelling. Nonetheless, the general trend of cut-off frequency values still indicates the occupied band, and the change-points between the bands are clearly recovered as well.

Instead of having only a finite number of frequency components, each of the three pieces of the signal is now constituted as a full bandpass signal. The results, shown on Fig. 3 are similar to those obtained with a large but finite number of sinusoids. This suggests the robustness of the algorithm to the accuracy of the model, given that the model is only a second order.

In the previous experiments, the signal did not contain any frequency component except of those in the bands mentioned, resulting in a noise-free analysis. We now add different amounts of noise by generating white noise and limiting its spectrum to the $1-25 \mathrm{~Hz}$ band. A case for SNR $=6 \mathrm{~dB}$ is shown on figure 4 . Figure 5 shows the effect of different levels of the noise on the mean values and standard deviations of the estimated cut-off frequencies for the signal in figure 3 It is clear that the noise perturbations significantly affect the accuracy and stability of the results. However, for relatively high SNR the results could still be acceptable for different applications.

To get an idea on how the performance of the proposed parametric method compares to standard non-parametric approaches, we compared our algorithm with STFT. We applied 

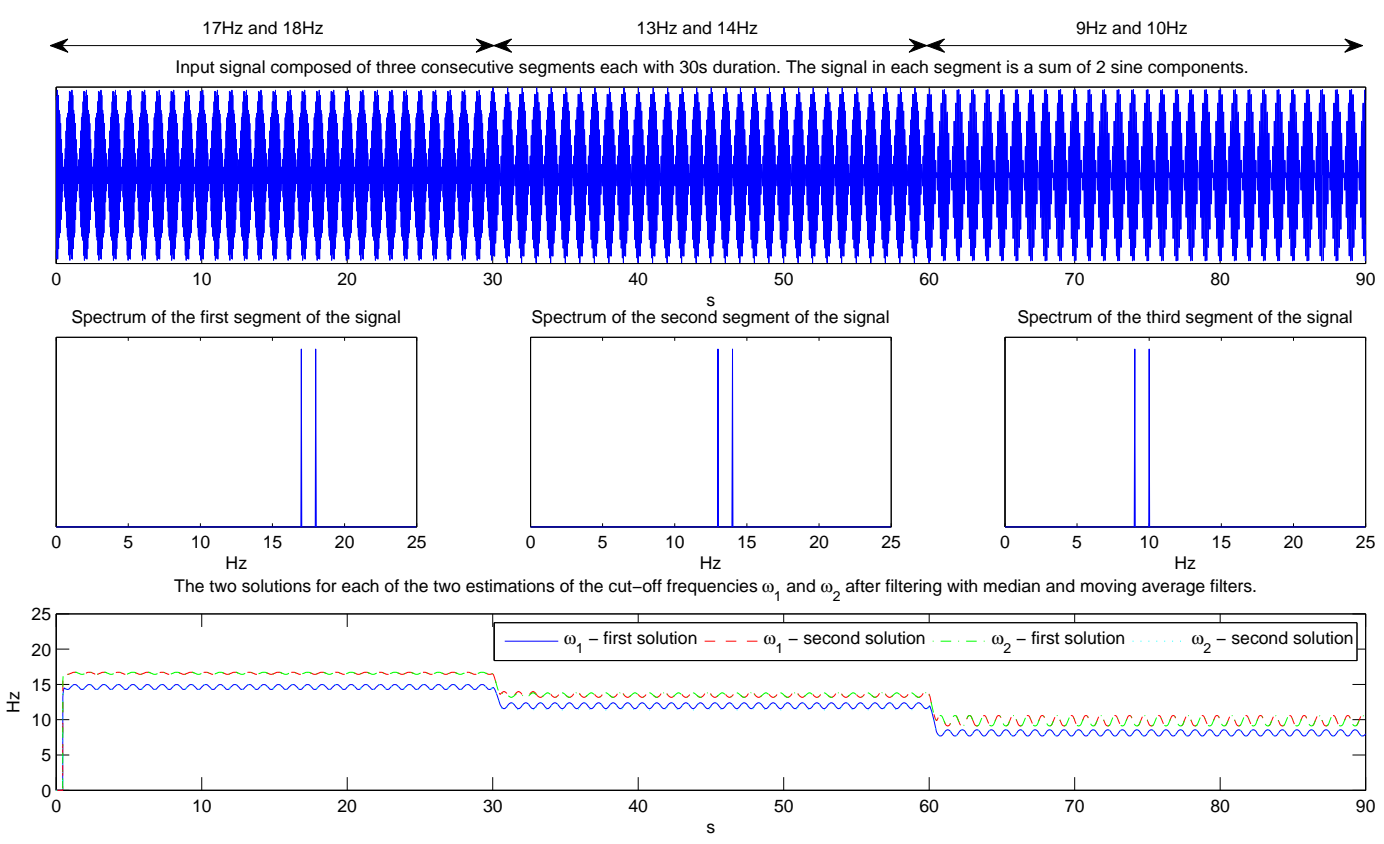

Fig. 1. Estimation results for piecewise signal with each segment composed of 2 sine functions.
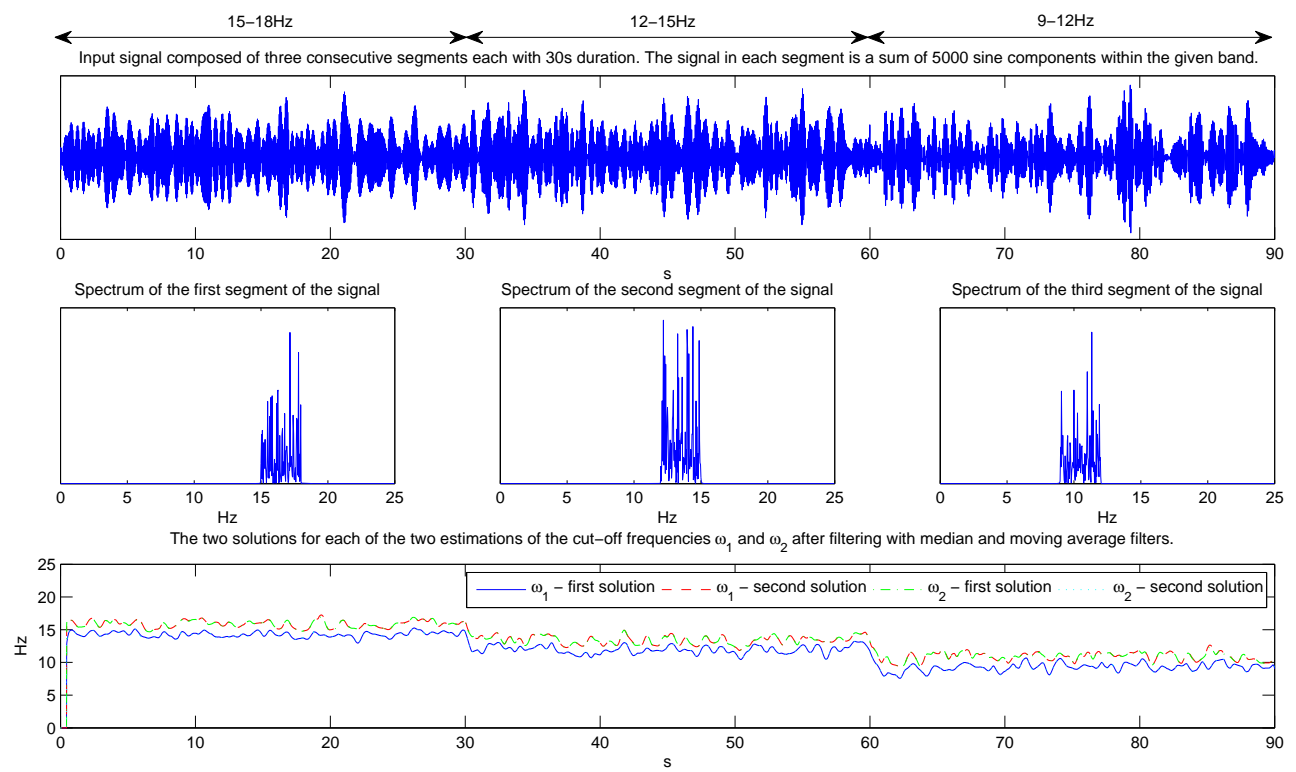

Fig. 2. Estimation results for piecewise signal with each segment composed of 5000 sine functions.

STFT on the noisy input signal shown on the first plot of figure 4. We set the window length for STFT to 90 samples, the same as the total length of the filters used with the proposed method. The last plot on figure 4 displays the result of STFT. It is clear that, even though the price for good time resolution is payed by lower spectral resolution, the non-parametric approach gives useful results as well. Change points appear clearly (no false negatives), which is however not the case for the cut-of frequencies. In on-line applications, large errors in estimation of the cut-of frequencies could cause false alarms in detection of change points (false positives). The results suggest that this is as well the case for the proposed approach.
We explore now the influence of the free parameters $M$ and $\nu$ on the estimation accuracy. Increasing $M$ means increasing the impulse length of the filters $h_{i, m}$. This results in higher sensitivity of the algorithm so that even the frequency components of the lower power can be detected. In general the estimated values of the frequency bands are wider than with lower $M$. This is demonstrated on figure 6 which is created using the same signal as figure 5 , but with $M$ set to 30 instead of 10 . The increase of $\nu$ has a similar effect: it results in wider effective impulse length of the filters (the number of the samples significantly different from 0 ). The results for $\nu=6$ are shown on figure 7 . 

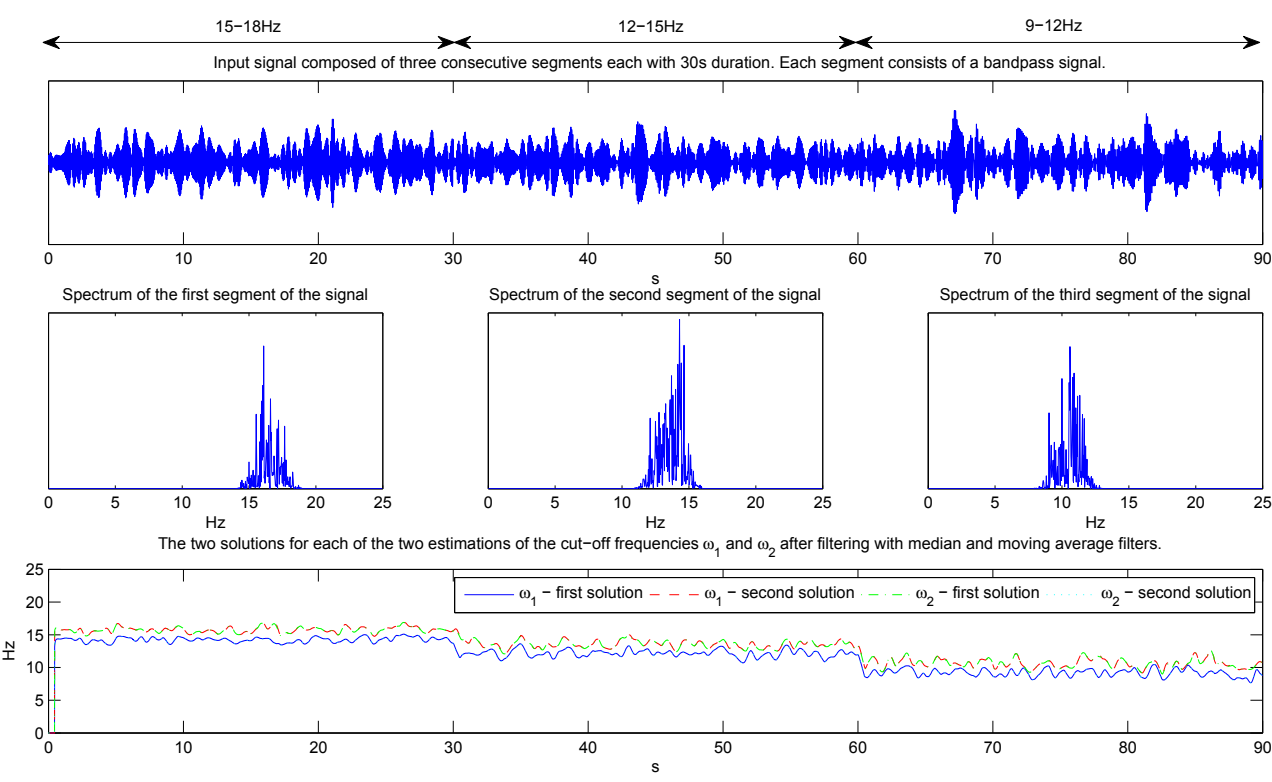

Fig. 3. Estimation results for piecewise bandpass signal.
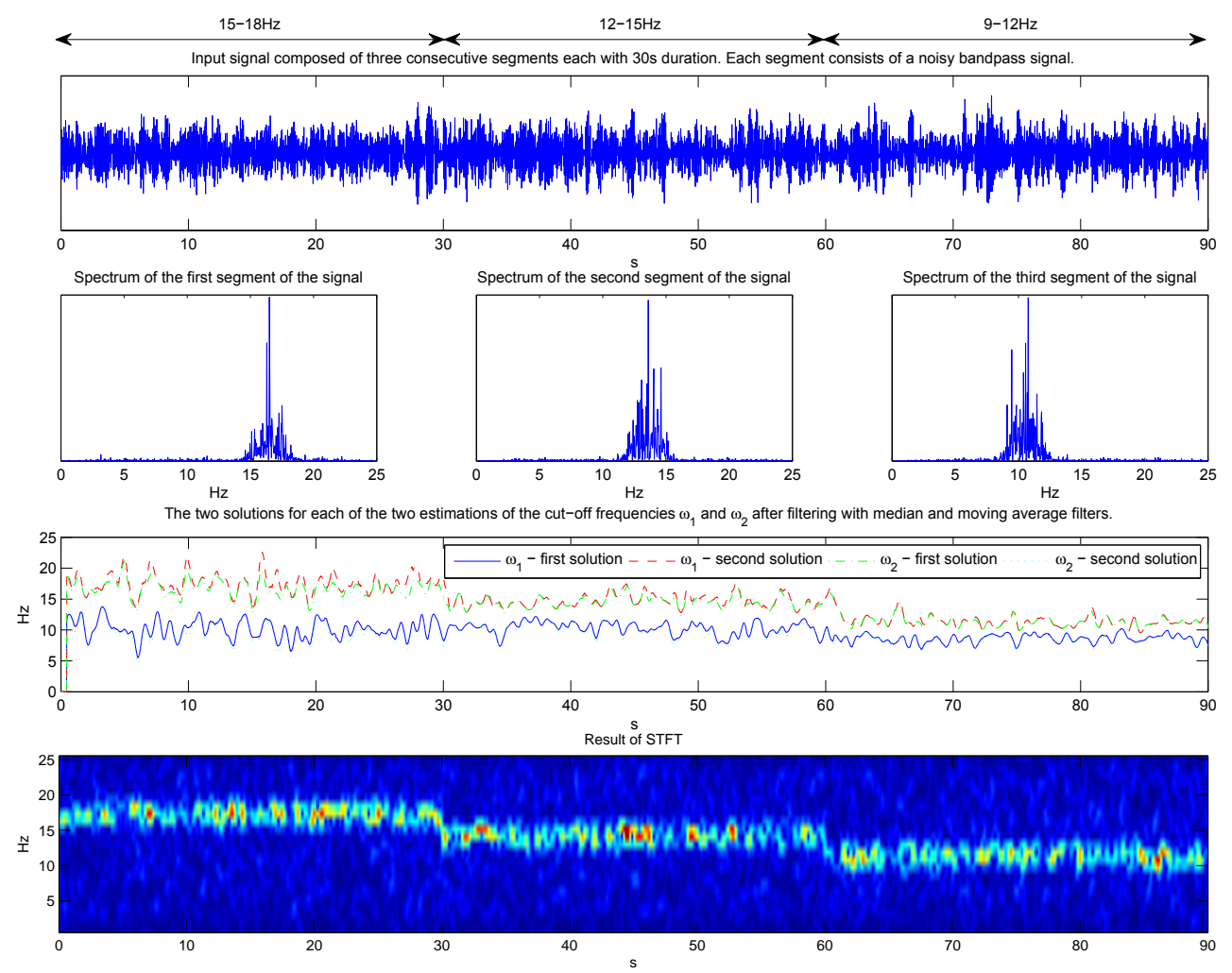

Fig. 4. Estimation results for noisy piecewise bandpass signal. $\mathrm{SNR}=6 d B$.

\section{CONCLUSION}

Even though we modeled the signal by a simple second order model, the estimated cut-off frequencies were meaningful even for the bandpass signal. Moreover, the estimations were meaningful even in the presence of a moderate amount of noise. We also showed that the sensitivity of the algorithm can be experimentally tuned by adjusting the free parameters
$M$ and $\nu$.

\section{ACKNOWLEDGMENT}

This work was supported by the foundation EADS and PHC COGITO project. 

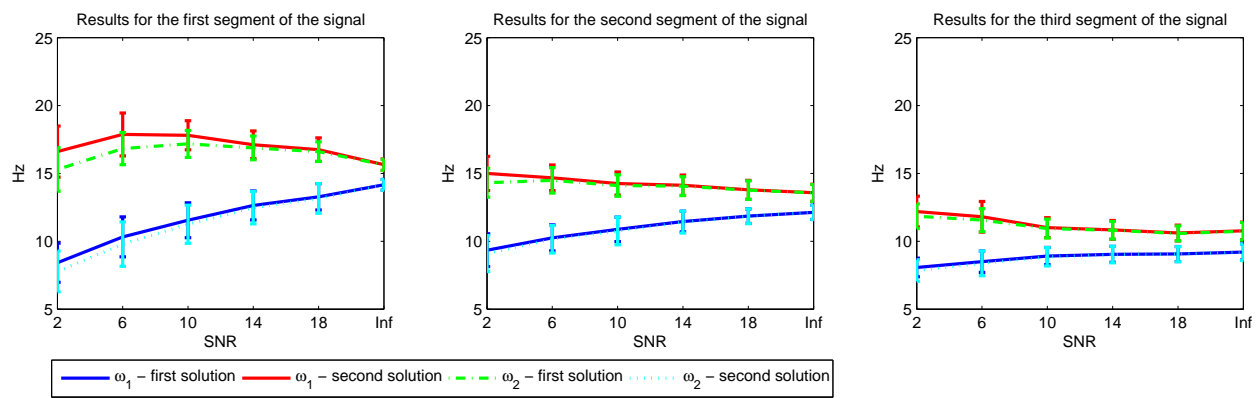

Fig. 5. Mean values of the estimated cut-off frequencies for each of the three segments for different SNR (in dB). Vertical bars denote the standard deviation of the estimated cut-off frequencies.
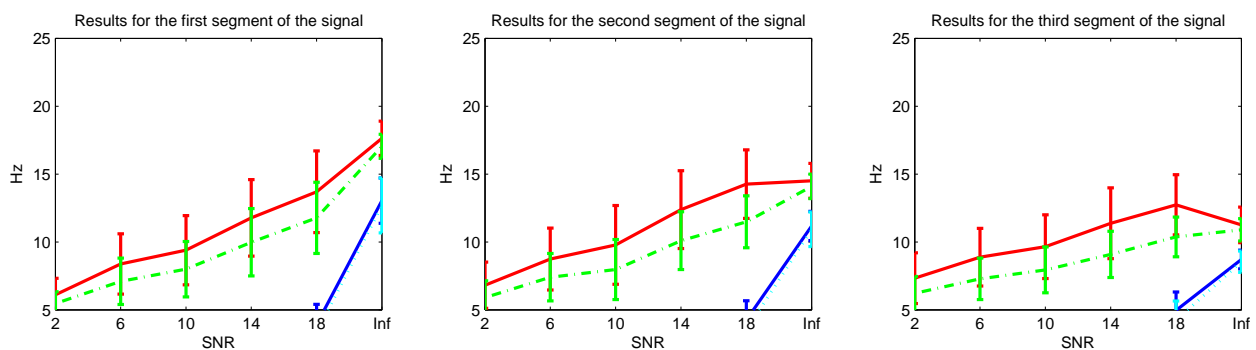

Fig. 6. Analysis of influence of parameter $M$. Simulation setup is similar to Fig. 5 but the value of $M$ is set to 30 instead of 10 .
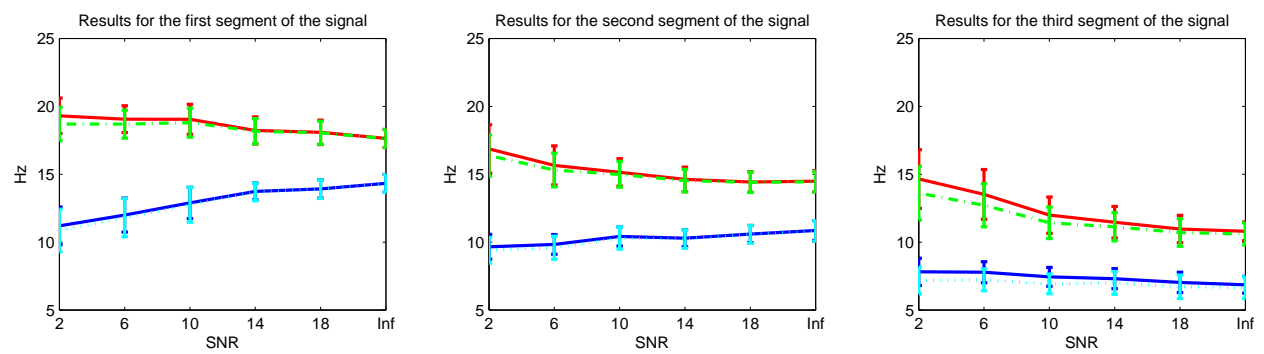

Fig. 7. Analysis of influence of parameter $\nu$. Simulation setup is similar to Fig. 5 but the value of $\nu$ is set to 6 instead of 3 .

\section{REFERENCES}

[1] M. Basseville and I. V. Nikiforov, Detection of Abrupt Changes: Theory and Application. Prentice-Hall, 1993.

[2] M. Mboup, "A Volterra filter for neuronal spike detection," ALIEN Inria, Research Report, 2008. [Online]. Available: http://hal.inria.fr/ inria-00347048

[3] M. Fliess, C. Join, and M. Mboup, "Algebraic change-point detection," App. Alg. in Eng., Com. \& Comp., vol. 21, pp. 131-143, 2010.

[4] M. Mboup, C. Join, and M. Fliess, "A delay estimation approach to change-point detection," in MED08, Ajaccio, France, 2008. [Online]. Available: http://hal.inria.fr/inria-00179775

[5] S. Rezk, C. Join, S. El Asmi, M. Dogui, and M. Hédi Bedoui, "Frequency change-point detection in physiological signals : an algebraic approach," Int. J. on Sci. and Tech. of Aut. Cont. \& Comp. Eng., vol. 2, no. 1, pp. 456-468, 2008.

[6] Z. Tiganj and M. Mboup, "Spike detection and sorting: Combining algebraic differentiations with ica," in Independent Component Analysis and Signal Separation, 8th Int. Conf., 2009, pp. 475-482.

[7] Z. Tiganj, M. Mboup, C. Pouzat, and L. Belkoura, "An algebraic method for eye blink artifacts detection in single channel eeg recordings," in 17th Int. Conf. on Biomagnetism, vol. 28, no. 6, 2010, pp. 175-178.

[8] B. Litt and J. Echauz, "Prediction of epileptic seizures," The Lancet Neurology, vol. 1, no. 1, pp. 22-30, 2002.

[9] G. Pfurtscheller and F. H. Lopes Da Silva, "Event-related eeg/meg synchronization and desynchronization: basic principles." Clinical Neurophysiology, vol. 110, no. 11, pp. 1842-1857, 1999.
[10] L. Kiltz, M. Mboup, and J. Rudolph, "Fault diagnosis on a magnetically supported plate," in Submitted to ICSCS, 2012.

[11] A. M. Ali, C. Join, and F. Hamelin, "Fault diagnosis without a priori model," Systems and Control Letters, vol. 61, no. 2, pp. 316-321, 2012.

[12] D. M. Himmelblau, Fault detection and diagnosis in chemical and petrochemical processes. Elsevier Scientific Pub. Co. New York, 1978.

[13] O. S. Salawu, "Detection of structural damage through changes in frequency: A review," Eng. Struct., vol. 19, no. 9, pp. 718-723, 1997.

[14] D. M. Himmelblau, Process analysis by statistical methods. Wiley New York, 1970.

[15] M. Fliess and H. Sira-Ramírez, "An algebraic framework for linear identification," in ESAIM: COCV. SMAI, 2003, vol. 9, pp. 151-168.

[16] A. Neves, M. Miranda, and M. Mboup, "Algebraic Parameter Estimation of Damped Exponentials," in EUSIPCO, Poznan, Pologne, 2007, pp. 965-969. [Online]. Available: http://hal.inria.fr/inria-00179732

[17] M. Mboup, "Parameter estimation for signals described by differential equations," Applicable Analysis, vol. 88, pp. 29-52, 2009.

[18] R. Ushirobira, W. Perruquetti, M. Mboup, and M. Fliess, "Algebraic parameter estimation of a biased sinusoidal waveform signal from noisy data," in Sysid 2012, 16th IFAC Symposium on System Identification, Brussels, Belgique, 2012. [Online]. Available: http: //hal.inria.fr/hal-00685067 